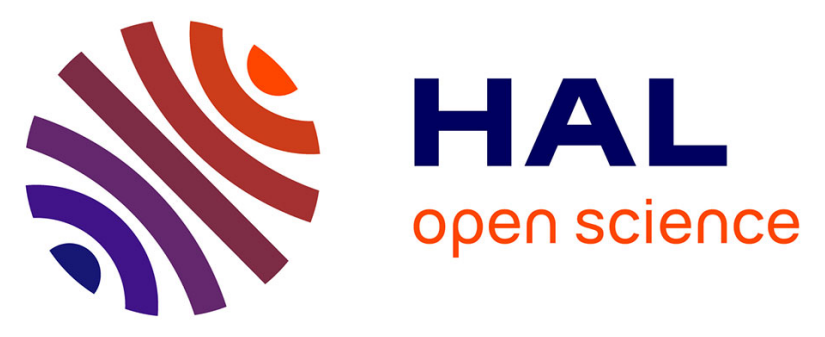

\title{
Sub-100 nanometer silver doped gold-cysteine supramolecular assemblies with enhanced nonlinear optical properties
}

Hussein Fakhouri, Martina Perić, Franck Bertorelle, Philippe Dugourd, Xavier Dagany, Isabelle Russier-Antoine, Pierre-Francois Brevet, Vlasta

Bonačić-Koutecký, Rodolphe Antoine

\section{To cite this version:}

Hussein Fakhouri, Martina Perić, Franck Bertorelle, Philippe Dugourd, Xavier Dagany, et al.. Sub-100 nanometer silver doped gold-cysteine supramolecular assemblies with enhanced nonlinear optical properties. Physical Chemistry Chemical Physics, 2019, 21 (23), pp.12091-12099. 10.1039/c9cp00829b . hal-02367612

\section{HAL Id: hal-02367612 \\ https://univ-lyon1.hal.science/hal-02367612}

Submitted on 28 Oct 2020

HAL is a multi-disciplinary open access archive for the deposit and dissemination of scientific research documents, whether they are published or not. The documents may come from teaching and research institutions in France or abroad, or from public or private research centers.
L'archive ouverte pluridisciplinaire HAL, est destinée au dépôt et à la diffusion de documents scientifiques de niveau recherche, publiés ou non, émanant des établissements d'enseignement et de recherche français ou étrangers, des laboratoires publics ou privés. 


\section{Sub-100-nanometer Silver Doped Gold-Cysteine Supramolecular Assemblies with Enhanced Nonlinear Optical Properties}

Received 00th January 20xx, Accepted 00th January 20xx DOI: $10.1039 / \times 0 \times x 00000 x$

\author{
Hussein Fakhouri, ${ }^{1}$ Martina Perić, ${ }^{2}$ Franck Bertorelle, ${ }^{1}$ Philippe Dugourd,${ }^{1}$ Xavier Dagany, ${ }^{1}$ Isabelle \\ Russier-Antoine, ${ }^{1}$ Pierre-François Brevet, ${ }^{1}$ Vlasta Bonačić-Koutecký, ${ }^{2,3}$ and Rodolphe Antoine ${ }^{1 *}$
}

\section{INTRODUCTION}

Supramolecular nanoarchitectures self-assembled from gold thiolate -Au-SR- polymers $^{1}$ have attracted much attention due to their high morphological variation. ${ }^{2,3}$ Indeed, the uniqueness of such nanomaterials resides in their multiple-type and multiple-strength weak interactions, such as Au-S coordination, aurophilic interactions between $\mathrm{Au}(\mathrm{I})$, and inter-ligand interactions including $\mathrm{H}$-bonding, electrostatic interactions and hydrophobic interactions. Because of the interplay of these interactions, nanomaterials can self-assemble into hierarchical structures. In particular, by using cysteine as the thiolate compound, the synergetic interplay of the electrostatic interaction between -cysteine side chains and the $\mathrm{Au}(\mathrm{I}) \ldots \mathrm{Au}(\mathrm{I})$ aurophilic attraction in the $\mathrm{Au}(\mathrm{I})$-Cys polymeric backbone may result in some helicity. ${ }^{4-6}$ On the other hand, under controlled external parameters (temperature, $\mathrm{pH} \ldots$...) $\mathrm{Au}(\mathrm{l})$-cysteine polymers can further assemble into bulky precipitate with lamellar structures. ${ }^{7}$ This capability to self-assemble into hierarchical structures leads also to unusual structure-optical

${ }^{1}$ Univ Lyon, Université Claude Bernard Lyon 1, CNRS, Institut Lumière Matière, UMR5306, Lyon, France. rodolphe.antoine@univ-lyon1.fr

${ }^{2}$ Center of Excellence for Science and Technology-Integration of Mediterranean Region (STIM) at Interdisciplinary Center for Advanced Sciences and Technology (ICAST), University of Split, Poljička cesta 35, 21000 Split, (Croatia)

${ }^{3}$ Chemistry Department, Humboldt University of Berlin, Brook-Taylor-Strasse 2, 12489 Berlin (Germany),vbk@chemie.hu-berlin.de.

Electronic Supplementary Information (ESI) available: [Size distributions for NPs in aqueous solutions obtained by dynamic light scattering. Quantitative XPS data and spectra. UV-Vis absorption spectra of $\alpha$-Au-Cys as a function of $\mathrm{pH}$. TPEF spectra of Ag-doped Au-Cys NPs aqueous solution. ) HRS line intensity for NPs in aqueous solution. TDDFT OPA and TPA spectra for model structures.]. See DOI: $10.1039 /$ x0xx00000x property relationship. ${ }^{4,7}$ Usually, a strong absorption band in the near UV range for $\mathrm{Au}(\mathrm{I})$-thiolates supramolecular assemblies is attributed to a ligand-to-metal charge transfer (LMCT) mixed with ligand-to-metal-metal charge transfer (LMMCT) excitation. ${ }^{4}$ The high order in $\mathrm{Au}(\mathrm{I})$-thiolates supramolecular structures is a key factor for the aurophilic effect, which in turn provides luminescent properties. ${ }^{8,9}$ Furthermore, due to the inherent chirality of cysteine molecules, cysteine $\mathrm{Au}(\mathrm{I})$-supramolecular assemblies display circular dichroism (CD) and constitute efficient targets for biosensing in particular for enantiomeric drug analysis. ${ }^{6}$

The investigation of the nonlinear optical (NLO) properties of silver and gold thiolated supramolecules is still in its infancy, and only few reports on NLO measurements have been reported. 5 , 10,11 We have recently shown that, although non-fluorescent through one-photon excitation, gold-cysteine supramolecular assemblies present two-photon excited fluorescence. They also exhibit second harmonic generation. Between large plasmonic gold metal nanoparticles (NPs), ${ }^{12}$ gold NPs hybrids ${ }^{13,14}$ and gold quantum nanoclusters NLO-phores, ${ }^{15,} 16$ chiral supramolecular gold-cysteine assemblies constitute therefore a new class of nanomaterials with favorable NLO properties driven by their supramolecular nanoarchitecture. Nevertheless, the exact supramolecular architecture of these systems remains a key feature to design efficient NLO systems. Indeed, centrosymmetry plays a major role in bestowing quadratic NLO properties for instance. It has been shown for example that the first hyperpolarizability of ligated-Au $\mathrm{A}_{25}$ clusters is strongly enhanced with silver alloying. ${ }^{17}$ Furthermore, both one- ${ }^{18}$ and two-photon ${ }^{19}$ excited fluorescence of ligated gold nanoclusters 

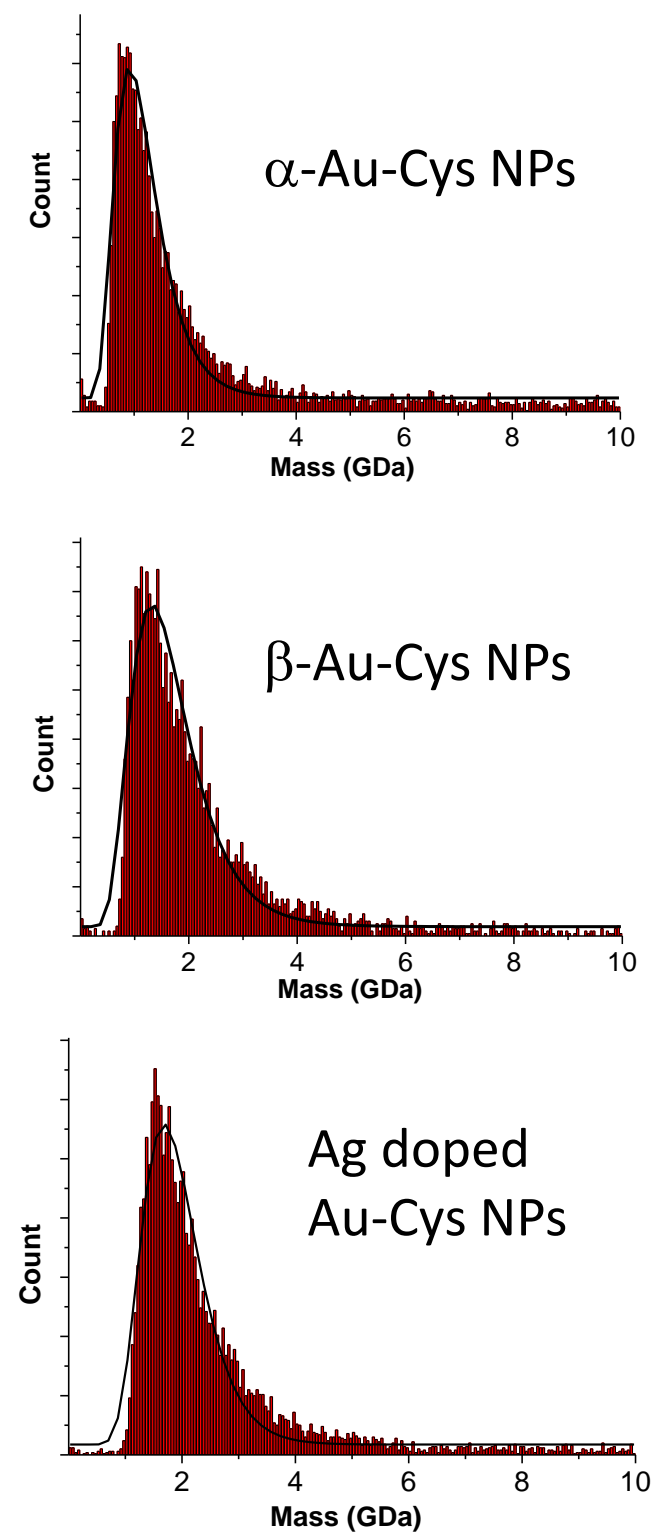

Figure 1: Mass histograms for the 3 samples $\alpha$-Au-Cys, $\beta$-AuCys, and Ag-doped Au-Cys NPs, obtained from chargedetection mass spectrometry (CDMS) measurements. Experimental distributions are fitted with lognormal distributions. The corresponding full widths at half maximum are $0.8,1.1$ and $1 \mathrm{GDa}$ for $\alpha$-Au-Cys, $\beta$-Au-Cys, and Ag-doped Au-Cys NPs respectively.

can be dramatically enhanced by alloying of the core with silver atoms.

For confirmed structure-optical property relationship, a control of the supramolecular architectures is required. However, synthetic control over the exact supramolecular architecture is usually difficult. Indeed, the spontaneous interactions of a gold salt with cysteine result in the formation of non-controllable self-assembly structures that can reach micrometer size range. ${ }^{20}$ In this work, we describe conditions for the controlled synthesis of sub-100-nanometer gold-cysteine and silver doped gold-cysteine supramolecular assemblies. We propose a twostep process involving (i) the reduction of $A u(I I I)$ ions to $A u(I)$ ions by cysteine (Cys) and the formation of polymeric structures of the form -[Cys-Au(I) $]_{n}-$ at high $\mathrm{pH}$ in the presence of trimethylamine (TEA), and (ii) acidic conditions allowing for the formation of helical supramolecular assemblies with a size range lower than $100 \mathrm{~nm}$. We also explore a silver-doping strategy to enhance the linear and nonlinear optical properties of gold-cysteine supramolecular assemblies. By an accurate characterization of the as-synthesized NPs (in particular thanks to their molar mass measurements), we have been able to measure for the first time, their absolute two-photon absorption and two-photon excited fluorescence cross-sections and their first hyperpolarizabilities. Huge values are obtained for silver doped gold-cysteine supramolecular assemblies, as compared to their corresponding undoped counterparts. In addition, we employ DFT and TD-DFT methods to study the geometric and electronic structures of model gold-cysteine as well as silver doped gold-cysteine compounds in order to address the structure-linear/nonlinear optical property relationship in order to gain insights into the origin of the nonlinear optical enhancement of silver-doped gold supramolecular assemblies.

\section{RESULTS AND DISCUSSION}

Gold-cysteine and silver doped gold-cysteine supramolecular architectures and their characterization

The self-assembly of chiral $-[\mathrm{Cys}-\mathrm{Au}(\mathrm{I})]_{n}-$ supramolecular assemblies have already been reported in the literature ${ }^{4,6,7}$ and usually involves a one-step simple reaction of $\mathrm{HAuCl}_{4}$ with $\mathrm{D}$ Cys, L-Cys, or D/L-Cys at $\mathrm{pH}$ values ranging between 3 and 7 , a $\mathrm{pH}$ where Cys side chain groups are in their zwitterionic form. Cys is a mild reducing agent for the reduction of $\mathrm{Au}(\mathrm{III})$ ions to $\mathrm{Au}(\mathrm{I})$ ions. It subsequently complexes with $\mathrm{Au}(\mathrm{I})$ to form polymeric helical structures of the form $-[\mathrm{Cys}-\mathrm{Au}(\mathrm{I})]_{\mathrm{n}}-$ with a diameter larger than $500 \mathrm{~nm} .{ }^{4,6,7}$ In this work, the gold-cysteine as well as silver doped gold-cysteine supramolecular assemblies are produced with a different mechanism leading to smaller particles with a diameter range lower than $100 \mathrm{~nm}$ (see below). We propose a two-step process for self-assembly and characterization of gold-cysteine and silver doped gold-cysteine supramolecular assemblies leading to helical nanostructures. Step (i) : The $A u(I I)$ ions are first reduced by Cys to $A u(I)$ ions and subsequently form polymeric $-[\mathrm{Cys}-\mathrm{Au}(\mathrm{I})]_{n}-$ structures at high $\mathrm{pH}$, where amine side chains are in their neutral form. Due to the presence of triethylamine (TEA) leading to an ion-pairing with the Cys carboxylic groups and thus some steric hindrance, the formation of helical structures is avoided. Note that we measured optical spectra during this first step. Absorption spectra (displayed in Fig. S1 in SI) do not show any band at 350 $\mathrm{nm}$ which is Indicative of the absence of helical motifs formed during the first step with TEA addition. Step (ii) : with the addition of acetic acid, the $\mathrm{pH}$ drops to $\sim 4$, and TEA molecules are removed from the $-[\mathrm{Cys}-\mathrm{Au}(\mathrm{I})]_{\mathrm{n}}-$ polymeric structures, allowing for the formation of helical structures. The presence of 
TEA in the first step is possibly the key factor for producing NPs with a size range lower than $100 \mathrm{~nm}$.

Gold-cysteine (with two different morphologies, coined $\alpha$-AuCys NPs, $\beta$-Au-Cys NPs, see experimental section) and silver doped gold-cysteine supramolecular assemblies were characterized by different techniques. We have obtained molar mass information for the supramolecular assemblies samples diluted in a water-methanol mixture $(50: 50 \mathrm{v} / \mathrm{v})$. As mentioned in the experimental section, charge detection mass spectrometry (CDMS) ${ }^{21-23}$ measures the mass of individual ions thanks to the independent measurement of the $\mathrm{m} / \mathrm{z}$ ratio and the charge $z$ for thousands individual ions. Mass histograms for the 3 samples are depicted in Figure 1. $\alpha$-Au-Cys, $\beta$-Au-Cys, and Ag-doped Au-Cys NPs show an average mass of 1.1 GDa, 1.5 GDa and $1.8 \mathrm{GDa}$ respectively. Of note, the accurate and direct measurement of the molar mass for supramolecular assemblies allows a quantitative determination of the NPs solution concentrations, an essential requisite for the absolute measurements of the TPA cross section and the first hyperpolarisability.

Figure S2 shows the size distributions for $\alpha$-Au-Cys, $\beta$-Au-Cys, and Ag-doped Au-Cys NPs in aqueous solutions obtained by dynamic light scattering (DLS). Only one component was observed in all cases, with a mean hydrodynamic diameter (HD) of $71 \mathrm{~nm}, 62 \mathrm{~nm}$, and $102 \mathrm{~nm}$ for $\alpha$-Au-Cys, $\beta$-Au-Cys, and Agdoped Au-Cys NPs respectively. The average mass extracted from CDMS spectra can be converted to an average NP diameter using the spherical shape approximation and an average density of Au-Cys nanoparticles of $\sim 3$ (by averaging density considering 19.3 for gold and $\sim 1.3$ for cysteine densities). Thus, $\alpha$-Au-Cys, $\beta$-Au-Cys, and Ag-doped Au-Cys NPs show an average estimated diameter of $106 \mathrm{~nm}, 119 \mathrm{~nm}$, and $125 \mathrm{~nm}$ respectively (in fair agreement with DLS measurements).

Quantitative XPS measurements confirmed that the Au: Cys ratio is close to a 1:1 stoichiometry in $\alpha$-Au-Cys, $\beta$-Au-Cys, and Ag-doped Au-Cys NPs (see Table S1 and Figure S3). We also used XPS to investigate the valence state of the gold atoms in the NPs. XPS spectra of $20 \mathrm{~nm}$ colloidal $A u(0)$ NPs standard and polymeric GS-Au(I) thiolates (where GS stands for glutathione) were obtained. They exhibit the Au $4 f 7 / 2$ binding energies (BE) at 83.8 and $85.0 \mathrm{eV}$, respectively. ${ }^{24}$ As shown in Figure S4, Au $4 \mathrm{f7} / 2$ binding energy (BE) for the three NPs samples is close to that of $\mathrm{Au}(\mathrm{I})(\mathrm{BE} 85.0 \mathrm{eV}$ ) in polymeric GS-Au(I) NPs, confirming the existence of $\mathrm{Au}(\mathrm{I})$ in $\alpha$-Au-Cys, $\beta$-Au-Cys, and $\mathrm{Ag}$-doped $\mathrm{Au}$ Cys NPs. Also in Figure S4, Ag $3 d$ binding energy (BE) curves confirm the presence of $\mathrm{Ag}$ only for Ag-doped Au-Cys NPs samples. High-resolution $\mathrm{Ag} 3 \mathrm{~d}$ binding energy (BE) for $\mathrm{Ag}$ doped Au-Cys NPs samples is close to that of $\mathrm{Ag}(\mathrm{I})$ (BE $374 \mathrm{eV}$ and $368.5 \mathrm{eV}$ ) in $\mathrm{AgNO}_{3},{ }^{20}$ suggesting the existence of $\mathrm{Ag}(\mathrm{I})$ in Ag-doped Au-Cys NPs (see Figure S4).

\section{Linear optical properties}

A UV-vis absorption peak (see Fig. 2a) is located at approximately 350-370 nm, a characteristic wavelength for transitions involving supramolecular gold (assigned to a ligandto-metal charge transfer (LMCT) mixed with ligand-to-metalmetal charge transfer (LMMCT) excitations). ${ }^{4}$ While the $\alpha$-AuCys NPs are found to be non-fluorescent in water, both $\beta$-AuCys and Ag-doped Au-Cys NPs have orange-red emission (maximum at $635 \mathrm{~nm}$ for $\beta$-Au-Cys and $670 \mathrm{~nm}$ for Ag-doped Au-Cys), large Stokes shift (>250 nm), and long fluorescence lifetime component ( $200 \mathrm{~ns}$ and $400 \mathrm{~ns}$ for $\beta$-Au-Cys Ag-doped Au-Cys NPs respectively). It was shown that $\beta$-Au-Cys NPs adopted lamellar like-assemblies and strongly interplay with each other ${ }^{7}$ leading to a kind of "stiffening" of the -(Au-Cys)chains by additional hydrogen bonding enabling fluorescence emission (as compared to non-fluorescent $\alpha$-Au-Cys NPs)..$^{25}$ The quantum yield (QY) of the strongest fluorescent species in water, i.e. Ag-doped Au-Cys NPs is $\sim 0.6 \%$, as determined by comparison with DCM (4(dicyanomethylene)-2-methyl-6-(pdimethylaminostyryl)-4H-pyran), see experimental section for details. However, the obtained QY value is probably underestimated because of the intensive light scattering of NPs in water. The stability of as-formed $\alpha$-Au-Cys NPs was explored as a function of $\mathrm{pH}$. The same absorption spectra are observed for $\mathrm{pH}$ values ranging from $\sim 5$ to 11 , confirming the stability of NPs over a large $\mathrm{pH}$ range. At low $\mathrm{pH}(<5), \alpha$-Au-Cys NPs might aggregate, leading to a distinct absorption spectrum (see fig. S5).
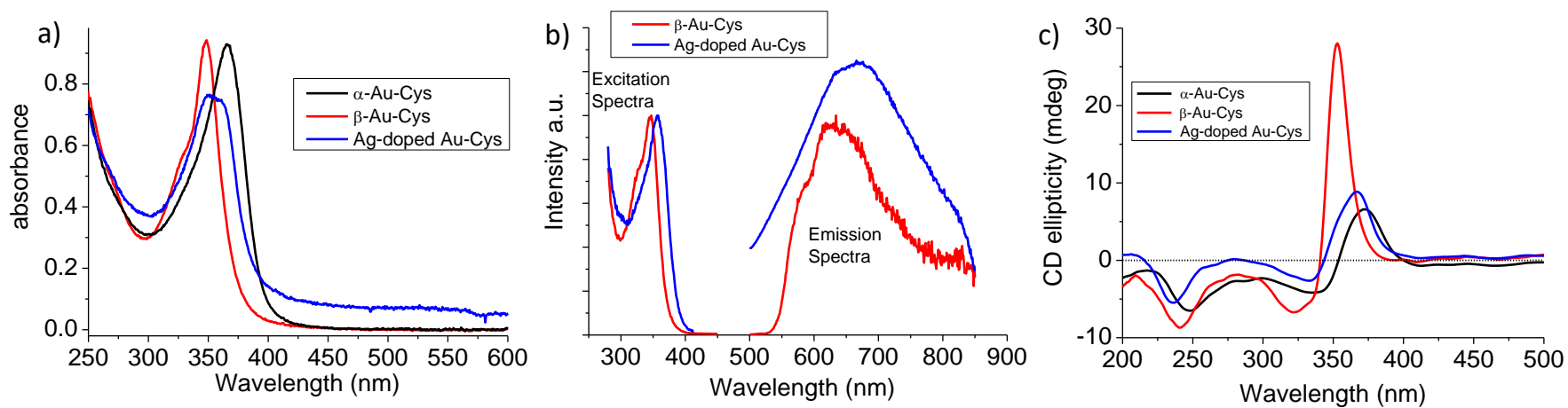

Figure 2: (a) UV-Vis absorption spectra of $\alpha$-Au-Cys, $\beta$-Au-Cys and Ag-doped Au-Cys prepared in water. (b) excitation and emission spectra of $\beta$-Au-Cys and Ag-doped Au-Cys NPs. The fluorescence of $\alpha$-Au-Cys is not measurable. The fluorescence intensities excited at $355 \mathrm{~nm}$ are plotted in arbitrary units. (c) CD spectra of $\alpha$-Au-Cys, $\beta$-Au-Cys and Ag-doped Au-Cys prepared in water. 
TABLE 1 : Absolute magnitude of the first hyperpolarizability, two-photon absorption (TPA) and two-photon excited fluorescence cross-sections (TPEF) for $\alpha$-Au-Cys, $\beta$-Au-Cys and Ag-doped Au-Cys NPs at several laser excitation wavelengths.

\begin{tabular}{|c|c|c|c|c|c|c|c|c|c|}
\hline & \multicolumn{4}{|c|}{ First hyperpolarizability $\left(\times 10^{-27}\right.$ esu) } & \multirow{2}{*}{$\begin{array}{l}\begin{array}{l}\text { TPA } \\
\text { cross } \\
\text { section } \\
\text { (GM) }\end{array} \\
720 \mathrm{~nm}\end{array}$} & \multicolumn{4}{|c|}{ TPEF cross section (GM) } \\
\hline & $720 \mathrm{~nm}$ & $750 \mathrm{~nm}$ & $800 \mathrm{~nm}$ & $850 \mathrm{~nm}$ & & $720 \mathrm{~nm}$ & $750 \mathrm{~nm}$ & $800 \mathrm{~nm}$ & $850 \mathrm{~nm}$ \\
\hline $\begin{array}{l}\alpha-A u-C y s \\
\text { NPs }\end{array}$ & 91 & 102 & 65 & & $6.310^{7}$ & & 1660 & 867 & \\
\hline $\begin{array}{l}\beta \text {-Au-Cys } \\
\text { NPs }\end{array}$ & 194 & 142 & 105 & & $6.310^{7}$ & & 2880 & 1780 & \\
\hline $\begin{array}{l}\text { Ag-doped } \\
\text { Au-Cys NPs }\end{array}$ & 476 & 356 & 225 & 208 & $12.910^{7}$ & 5430 & 3630 & 2400 & 1300 \\
\hline
\end{tabular}

The circular dichroism (CD) signals for $\alpha$-Au-Cys and Ag-doped Au-Cys NPs supramolecular assemblies present a positive band at $\sim 360 \mathrm{~nm}$, signature of the formation of a helical chiral structure..$^{4-6} \mathrm{~L}$-cysteine, a chiral natural amino-acid is used in this work. The structure suggesting chirality over some length scale might be due to the specific electrostatic interaction among the -SR side chains in the $\mathrm{Au}(\mathrm{I})$-Cys polymeric backbone, which likely results in some helicity. Interestingly, for $\beta$-Au-Cys, the CD signal is blue-shifted and increases by more than a fourfold factor as compared to the one recorded for $\alpha$-Au-Cys (see Fig. 2c). This band shift accompanied with a strong enhancement might be the signature of a structural change between $\alpha$-Au-Cys and $\beta$-Au-Cys as evidenced by Söptei et al. ${ }^{7}$ Indeed, upon heating, $\alpha$-Au-Cys NPs transform into $\beta$-Au-Cys NPs adopting a multilayer superlattice structure. ${ }^{7}$ Chiral effects might be enhanced with the aggregation of Au-Cys units into sheets and multilayers. Of note the chiral signal of Ag-doped Au-Cys NPs is very similar to the one observed for $\alpha$-Au-Cys (see Fig. 2c). Most likely, the addition of the silver salt does not alter the helical chiral structure.

Clearly, the addition of silver salt to $\alpha$-Au-Cys NP solution (1:20 $\mathrm{Au} / \mathrm{Ag}$ molar ratio) leads to a dramatic enhancement, from nonmeasurable fluorescence signals for $\alpha$-Au-Cys NPs to intense red emission (with QY 0.6 \%) for Ag-doped Au-Cys NPs. A smaller Au:Ag molar ratio $(<1: 20)$ leads to less fluorescent species (but with similar fluorescence spectra). On the other hand, increasing the $\mathrm{Au}: \mathrm{Ag}$ molar ratio $(>1: 20)$ leads certainly to the aggregation of $\mathrm{Ag}$ into small $\mathrm{Ag}$ clusters since a new band at $\sim 400 \mathrm{~nm}$ emerges which may be attributed to the signature of a plasmon band. Of note, NPs produced with Au:Ag molar ratio $>1: 20$ are less stable with time and tends to precipitate. From a structural point of view, the addition of the silver salt has led to the incorporation of $\mathrm{Ag}^{+}$ions in the -(Au-Cys)- chains. Two possible incorporation sites have to be considered: (i) external : an intramolecular chelation effect fostered by the formation of a five-membered chelation ring between the $\mathrm{COO}^{-}$and $\mathrm{NH}_{2}$ groups with $\mathrm{Ag}^{+}$, (ii) internal: $\mathrm{Ag}^{+} / \mathrm{Au}^{+}$substitution in the -(AuCys)- chain motifs. Of note, the external incorporation would lead to the interruption of intercysteine $\mathrm{H}$-bonds and thus the destruction of the helical chiral structure. ${ }^{26}$ The fact that the $\alpha$ Au-Cys and Ag-doped Au-Cys NPs CD signals are similar precludes this external incorporation scenario, and most likely -(Au-Cys)- chain motifs are randomly substituted by $\mathrm{Ag}^{+} / \mathrm{Au}^{+}$ within the polymer chain.

\section{Nonlinear optical properties}

The TPEF spectra with excitation ranging between 720 and 850 $\mathrm{nm}$, were recorded for the supramolecular assemblies, as shown in Figure S6. For TPEF, a broad band in the visible range between 350 and $600 \mathrm{~nm}$ centered at about $450 \mathrm{~nm}$ was

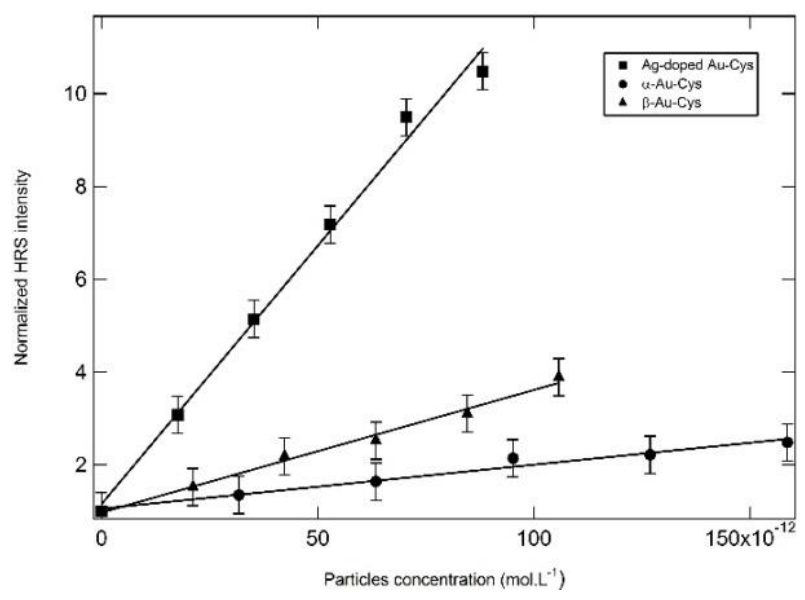

Figure 3 : Linear plots of the normalized HRS intensity for aqueous solutions of $\alpha$-Au-Cys, $\beta$-Au-Cys and Ag-doped AuCys NPs as a function of concentration for a $800 \mathrm{~nm}$ fundamental laser excitation. The continuous lines correspond to the linear adjustments to the experimental data. 
observed. A similar spectrum was obtained for the $\mathrm{Au}_{10}(\mathrm{SG})_{10}$ catenane (SG stands for glutathione) nanoclusters. ${ }^{27} \mathrm{Au}_{10}(\mathrm{SG})_{10}$ is a cyclic polymeric -(Au-SG)- chain-like structure. Calculations showed that the leading excitations within the S1 state involve $\mathrm{Au} \cdots \mathrm{Au}$ units together with neighboring sulfur atom subunits. ${ }^{27}$ Table 1 shows the TPEF cross-sections in GM units for Au-Cyst and $\mathrm{Ag}$ doped Au-Cyst supramolecular assemblies in the 720$850 \mathrm{~nm}$ wavelength range. TPEF cross-sections exceed $1000 \mathrm{GM}$ and reach $5430 \mathrm{GM}$ for Ag-doped Au-Cys NPs at $720 \mathrm{~nm}$, following the trend TPEF( $\alpha-A u-C y s)<\operatorname{TPEF}(\beta-A u-C y s)<\operatorname{TPEF}(\mathrm{Ag}-$ doped Au-Cys). Also for each NP, the TPEF cross-section increases as the laser wavelength excitation decreases. This behavior is due to a resonance effect. ${ }^{28}$ Indeed, below $800 \mathrm{~nm}$, the two-photon excitation step can reach the electronic state responsible for the absorption band at $\sim 360 \mathrm{~nm}$. Both TPA and TPEF cross-sections are dramatically larger than those reported for Au NCs (<1 GM). ${ }^{27,} 29$ These sub-100 nm supramolecular assemblies are therefore potentially interesting for multiphoton applications.

The first hyperpolarizability of the as-prepared supramolecular assemblies was obtained using the hyper-Rayleigh scattering (HRS) technique. ${ }^{29}$ The HRS intensity was recorded for several concentrations of the NPs dispersed in aqueous solutions (Figure S7 and Figure 3) and short-range spectra were recorded around the HRS wavelength. The subtraction of the fluorescence background was performed as described in our previous works. ${ }^{29}$ Table 1 also displays the first hyperpolarizability values in units of $10^{-27}$ esu for Au-Cyst supramolecular assemblies and $\mathrm{Ag}$ doped Au-Cyst supramolecular assemblies in the $720-850 \mathrm{~nm}$ wavelength range. Hyperpolarizabilities exceed $100 \times 10^{-27}$ esu and reach $476 \times 10^{-27}$ esu for Ag-doped Au-Cys NPs at $720 \mathrm{~nm}$ excitation. They follow the trend $\beta(\alpha-A u-C y s)<\beta$ ( $\beta$-Au-Cys) $<\beta$ (Ag-doped Au-Cys), similarly to the TPEF cross-section. Likewise, for each $N P$, the $\beta$ values increase as the excitation laser wavelength decreases. This behavior is is due to the resonance effect, similarly to the TPEF case. ${ }^{28}$ Finally, we would like to emphasize that the hyperpolarizability values reported here for Au-Cys NPs and Ag-doped Au-Cys NPs are in the same order of magnitude than the values reported for spherical plasmonic Au NPs with sizes in the range 50-100 nm (Au $50 \mathrm{~nm}: 124 \times 10^{-27}$ esu; Au 80 $\mathrm{nm}: 342 \times 10^{-27}$ esu: Au $100 \mathrm{~nm}: 668 \times 10^{-27}$ esu at $800 \mathrm{~nm}$ laser excitation, determined using the known first hyperpolarizability for pure water). ${ }^{12}$, 30 However, the hyperpolarizability for plasmonic NPs has a different origin as compared to that of supramolecular assemblies. Indeed, it has been demonstrated that the hyperpolarizability finds its origin from the breaking of centrosymmetry at the surface for plasmonic NPs. Here, for
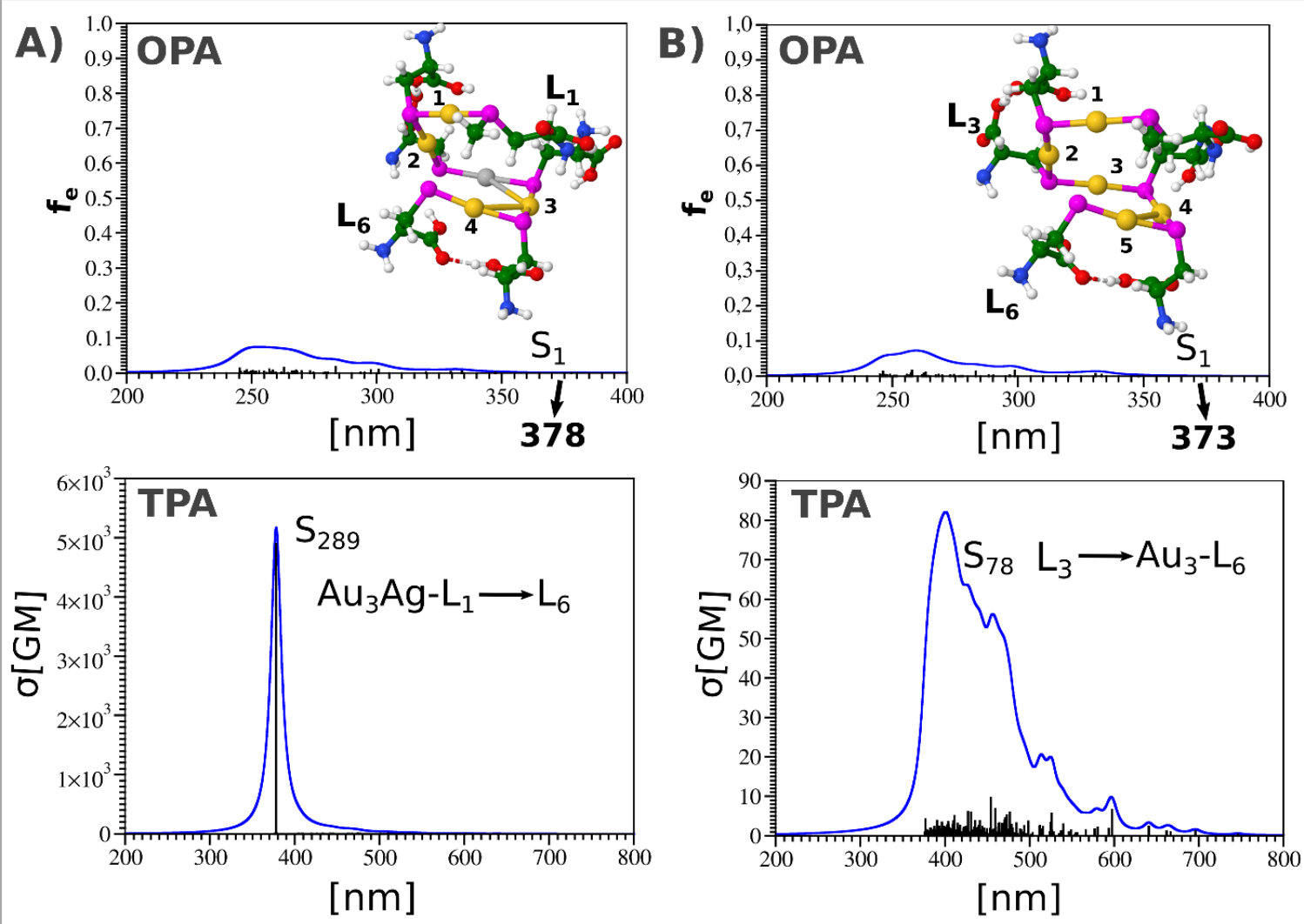

Figure 4: TDDFT (upper part) OPA and (lower part) TPA spectra for optimized structures of the model systems: $\mathrm{A}$ ) $\mathrm{Au}_{4}{ }^{-} \mathrm{Ag}^{-\mathrm{Cys}_{6}}{ }^{-}$ $\mathrm{CH}_{3}$ and $\mathrm{B}$ ) $\mathrm{Au}_{5}-\mathrm{Cys}_{6}-\mathrm{CH}_{3}$. Structure arts involved in the leading excitations of the $\mathrm{S}_{289}$ and $\mathrm{S}_{78}$ states are labeled according to the structures shown in the upper part. Broadening of the spectral lines is simulated with a Lorentzian profile with a half-width of $15 \mathrm{~nm}$ (blue lines). (Ag-grey, Au-yellow, S-magenta, C-green, O-red, N-blue, H-white). 
supramolecular assemblies, the origin of the hyperpolarizability may stem from the non-centrosymmetric molecular structure of the NP volume due the molecular structure and, also to chirality. The charge transfer nature of the -(Au-Cys)- motif as well as silver doping may then be key factors enhancing the first hyperpolarizabilities, as reported in this work.

\section{Insights from theoretical approach: structure-optical property relationship}

Two model systems for the experimentally prepared supramolecular assemblies have been considered, namely $\mathrm{M}_{3}-\mathrm{Cys}_{4}-\mathrm{CH}_{3}$ with $\mathrm{M}=\mathrm{Au}$ and $\mathrm{Ag}-\mathrm{Au}_{2}$ and $\mathrm{M}_{5}-\mathrm{Cys}_{6}-\mathrm{CH}_{3}$ with $\mathrm{M}=\mathrm{Au}_{5}$ and $\mathrm{Ag}-\mathrm{Au}_{4}$ in order to determine the role of the silver atoms as well as the transition from the smallest subunit to larger subunits for which helical structure is supposed to occur. Notice that the $\mathrm{CH}_{3}$ terminal group introduced in these model systems serves as an end-protection and provides an even number of electrons. In addition the ion-pairing of cysteines (zwitterionic form) in both model systems have been introduced by taking into account water solvent.

We focus on the influence of doping Au-Cys systems with silver atoms on the linear and nonlinear optical properties from a conceptual point of view. For all model systems, doping with silver atoms does not influence considerably the linear absorption properties OPA (one-photon absorption) due to the fact that excitations take place between molecular orbitals which are energetically very similar in pure Au-Cys and Ag doped Au-Cys. The OPA spectra for two isomeric structures of $\mathrm{Ag}$ doped and of pure $\mathrm{Au}$ $\mathrm{M}_{5}-\mathrm{Cys}_{6}-\mathrm{CH}_{3}$ (where $\mathrm{M}$ stands for the metal, either $\mathrm{Au}$ or $\mathrm{Ag}$ ) together with molecular orbitals responsible for one-photon transitions are shown in Figure 58 illustrating that silver atoms do not have influence on the OPA properties of the systems.

In contrast, the silver atoms play a key role for the nonlinear properties. The latter are several orders of magnitude larger in doped systems as compared to the pure undoped Au-Cys systems (cf. Figure 4 and Figure S10). OPA and TPA (two photon absorption) optical properties of helical model compounds are presented in Figure 4 and confirm the experimental findings, in particular that the huge enhancement of the nonlinear absorption is due to silver atom doping effect which induces large transition dipole moments. Notice that it is an open question to know whether doping by other noble metal atoms (e.g. $\mathrm{Cu}$ ) with similar energy ordering of orbitals, might cause similar effects or not. This might be important for design of new doped supramolecular assemblies inducing enhanced nonlinear properties.

Metal atoms as well as ligands are involved in transitions with large cross-sections. The resonance between one-photon and two-photon excited states at $377 \mathrm{~nm}$, in $\mathrm{Au}_{4}-\mathrm{Ag}-\mathrm{Cys}_{6}-\mathrm{CH}_{3}$ (see Fig. S9) is responsible for the blowing up of the TPA cross-section. The latter can be brought back to realistic value by introducing an adequate damping factor. ${ }^{31}$ It is interesting to notice that enlargement of the system may influence the TPA cross-section enhancement due to the Ag atom doping as shown by experimental findings. In contrast, for pure Au-Cys systems, no resonance between one-photon and two- photon excited states has been found. As a result, the two-photon cross-sections are very low.

The key role of $\mathrm{Ag}$ atom doping for two photon absorption has been also found for the smallest system $\mathrm{M}_{3}-\mathrm{Cys}_{4}-\mathrm{CH}_{3}$ which does not have a helical structure, as shown in Figure S10. The pure Au-Cys compound has a negligible TPA cross-section in comparison with the two isomers in which a Ag atom is present. In contrast, for linear optical absorption properties, the $\mathrm{Ag}$ atom does not have any substantial influence, as shown in Figure S11. Moreover, the role of the silver atom for the enhancement of the nonlinear properties remains unchanged in the smallest model system in comparison with the helical model system.

In summary theoretical findings support experimental results and allow for the generalization of single $\mathrm{Ag}$ atom doping in the context of the enhancement of the nonlinear optical properties of doped AuCys supramolecular assemblies. Moreover, the ion-pairing of cysteines in $\mathrm{Au}_{2}-\mathrm{Ag}-\mathrm{Cys}_{4}-\mathrm{CH}_{3}$ as well as in helical model system $\mathrm{Au}_{4}-$ $\mathrm{Ag}-\mathrm{Cys}_{6}-\mathrm{CH}_{3}$ has been illustrated by taking into account water solvent (cf. Computational section and Figure S12). For $\mathrm{Au}_{2}-\mathrm{Ag}-\mathrm{Cys}_{4}-\mathrm{CH}_{3}$ with ion-pairing of cysteines, according to TDDFT calculations, the resonance between OPA and TPA states occurs also confirming presence of large TPA cross-section.

Our model systems serve for three purposes: i) to show appearance of helical structure; ii) to illustrate similarities between gas phase and solvent and iii) to compare the quantitative influence of different functionals (cf. Computational).

\section{CONCLUSION}

In this work, we have proposed a simple and robust two-step process at elevated $\mathrm{pH}$ using trimethylamine for the selfassembly and formation of Au-Cyst supramolecular assemblies. Unlike many other reported works, involving a simple one-step process at $\mathrm{pH} \mathrm{3-7}$ and leading to sub $\mu \mathrm{m}$ nanoparticles, our synthesis leads to sub-100 nm supramolecular nanostructures. These nanostructures are found to be stable in a wide $\mathrm{pH}$ range (from $\sim 5$ to 11). Two strategies were proposed to enhance their nonlinear optical properties : (i) thermal heating that induces coiling, aggregation, and ultimately formation of lamellar structures and (ii) alloying of the NPs with silver atoms. Silverdoping leads to the dramatic enhancement of the optical properties of Au-Cyst supramolecular assemblies in the nonlinear optical regime. This has been confirmed by theoretical investigations of one and two-photon optical properties for model systems. Huge enhancement of the twophoton absorption cross section arises with the doping of AuCyst supramolecular assemblies by a single silver atom. These findings open new routes for the preparation of novel materials with enhanced nonlinear optical properties. The combination of strong circular dichroism activities along with enhanced emission properties in particular after two-photon absorption should enlarge the toolbox of supramolecular nanoarchitectures for bio-applications.

\section{EXPERIMENTAL AND CALCULATIONS}


Materials. All the chemicals were commercially available and used as received. Tetrachloroauric(III) acid trihydrate $\left(\mathrm{HAuCl}_{4} \cdot 3 \mathrm{H}_{2} \mathrm{O}\right)$ and silver trifluoroacetate were purchased from Acros Organics, L-cysteine, triethylamine (TEA) and glacial acetic acid, methanol $(\mathrm{MeOH})$, diethyl ether $\left(\mathrm{Et}_{2} \mathrm{O}\right)$ were purchased from Sigma-Aldrich. Ultrapure water with specific resistivity of 18.2 $\mathrm{M} \Omega$ was used throughout the synthesis.

Synthesis of $\boldsymbol{\alpha}$ - and $\boldsymbol{\beta}$-Au-Cys NPs. Briefly, $0.38 \mathrm{mmol}$ of cysteine was dissolved in $20 \mathrm{ml}$ of water and $0.5 \mathrm{ml}$ of triethylamine. $0.5 \mathrm{ml}$ of gold salt $(0.127 \mathrm{mmol}, 50 \mathrm{mg})$ was quickly added and the solution was stirred $30 \mathrm{~s}$ by inversion (the color becomes slightly yellow). Quickly, $1 \mathrm{ml}$ of glacial acetic acid was added to induce precipitation of $\alpha$-Au-Cys NPs which was centrifuged ( $5 \mathrm{mn} / 6000 \mathrm{rpm}$ ). The supernatant was removed and $\alpha$-Au-Cys NPs were redispersed in water $(2 \mathrm{ml}$ with $100 \mu \mathrm{l}$ of TEA, vortex time $5 \mathrm{mn}$ ). $20 \mathrm{ml}$ of methanol was then added to precipitate $\alpha$-Au-Cys NPs with centrifugation (10 mn/6000 $\mathrm{rpm})$. $\alpha$-Au-Cys NPs were also redispersed in $2 \mathrm{ml}$ of water with $10 \mu \mathrm{l}$ of TEA and precipitated with $\mathrm{MeOH}(10 \mathrm{ml}) / \mathrm{Et}_{2} \mathrm{O}(10 \mathrm{ml})$. After centrifugation $(10 \mathrm{mn} / 6000 \mathrm{rpm})$, the product was dried in air. For all experiments, the resulting powder was dissolved in water containing $0.1 \% \mathrm{v} / \mathrm{v}$ of TEA $\left(\mathrm{pH}^{\sim 11}\right)$. For producing $\beta$ Au-Cys NPs, the solution containing $\alpha$-Au-Cys NPs was heated at $70^{\circ} \mathrm{C}$ for $16 \mathrm{~h}$. It is thought that, upon heating, polymeric chains from $\alpha$-Au-Cys NPs coiled, aggregated, and ultimately form more compact lamellar sheets. ${ }^{7,} 32$

Synthesis of Silver-doped Au-Cys NPs. The synthesis of these NPs is made using 2 steps, at the first, the $\alpha$-Au-Cys NPs solution was used, we added to the solution silver trifluoroacetate (1:20 Au/Ag molar ratio) and the solution was first stirred for $24 \mathrm{~h}$. Then in a second step, the solution was heated at $70^{\circ} \mathrm{C}$ for 60 hours. After that the solution was purified following the same steps as described for $\alpha$-Au-Cys NPs.

Characterization. Charge-Detection Mass-Spectrometry (CDMS) is home-made instrument used to measure mass distribution; it extends the limit of other MS devices by measuring molar weights in the range of mega- to giga-dalton thus allowing the study of larger system such as viruses, ${ }^{33}$ bioparticles $^{22}$ and even NP assemblies. ${ }^{21,} 23$ Experiments were performed on a custom-built charge detection mass spectrometer with an electrospray ionization (ESI) source. This instrument is described in detail elsewhere. ${ }^{34}$ The samples were injected at flow rates of $0.2-0.6 \mathrm{~mL} / \mathrm{h}$, and entered the electrospray chamber through a $0.1 \mathrm{~mm}$ internal diameter stainless steel capillary tube located inside the needle tip. Nitrogen drying gas was injected between the end cap and the transfer glass capillary and flew through a heater typically set at $200^{\circ} \mathrm{C}$. The vacuum interface was composed of a glass transfer capillary that passes the ions into the first stage of the vacuum system, an end cap, a skimmer between the first and second vacuum stages, an hexapole ion guide and an exit lens. The charge detection device was used in a single pass mode. ${ }^{35}$ The signal induced on the tube was picked up by a JFET transistor and was amplified by a low-noise, charge-sensitive preamplifier and then shaped and differentiated by a home-built amplifier. The signal was recorded with a waveform digitizer card that recorded the entire waveform for each ion passing through the detector tube at a sampling rate of $10 \mathrm{MHz}$. The data were transferred to a desktop computer where they were analyzed to compute the charge and mass of each ion. Internal calibration in charge was performed using a test capacitor that allowed a known amount of charge to be pulsed onto the pickup tube. In addition, an external calibration was performed using NIST traceable size standards. ${ }^{21}$ Mass histograms are built from the collection of a statistically relevant number of single mass measurements for each sample (>2000 typically).

Hydrodynamic particle diameters were measured by dynamic light scattering (DLS) using a Malvern Zetasizer Nano ZS. A 633 $\mathrm{nm}$ wavelength laser beam was sent to a diluted sample maintained at $25^{\circ} \mathrm{C}$ and the scattered signal intensity was analyzed at a $173^{\circ}$ angle. The cumulant analysis method was used.

X-ray Photoelectron Spectroscopy (XPS) measurements were carried out using a PHI Quantera SXM instrument (Physical Electronics, Chanhassen, USA) equipped with a $180^{\circ}$ hemispherical electron energy analyzer and a monochromatized Al K $\alpha(1486.6 \mathrm{eV})$ source operated at $15 \mathrm{kV}$ and $4 \mathrm{~mA}$. The analysis spot had a diameter of $200 \mu \mathrm{m}$ and the detection angle relative to the substrate surface was $45^{\circ}$.

UV-visible, fluorescence and circular dichroism (CD) measurements. UV-vis spectra in solution were recorded using an AvaSpec-2048 fiber optic spectrometer and an AvaLight-DH$S$ deuterium-halogen light source. Fluorescence excitation and emission spectra were obtained using a Fluoromax-4 Horiba fluorimeter. With the same device (Fluoromax-4 Horiba), a complementary module allows the acquisition of fluorescence decay curve. A nano-LED excitation at $370 \mathrm{~nm}$ is used for the pulsed excitation with a duration of the order of ten picoseconds. Time Correlated Single Photon Counting with a resolution of $7 \mathrm{ps} /$ channel allows the measurement of lifetimes from $200 \mathrm{ps}$ to $0.1 \mathrm{~ms}$.

Quantum yield value is obtained using an external reference with a known quantum yield value. We used DCM (4(dicyanomethylene)-2-methyl-6-(p-dimethylaminostyryl)-4Hpyran), the quantum yield of this molecule is $43 \%$ and to calculate the $Q Y$ we used the following equation:

$$
\emptyset_{x}=\emptyset_{R e f} \times \frac{A_{R e f}}{A_{x}} \times \frac{F_{x}}{F_{R e f}} \times\left(\frac{n_{x}}{n_{R e f}}\right)^{2}
$$

where $\varnothing$ is the QY, A is the value of absorption, $F$ is the area of fluorescence spectra and is the refraction indices of the solvent used ( $x$ and Ref stand to NP's used and reference respectively). $C D$ spectra were recorded using a home-made setup based on Photo Elastic Modulator and lock-in amplifier detection devices previously described in Ref. ${ }^{36}$

Nonlinear optical measurements. The set-up for hyperRayleigh scattering (HRS) and two-photon emission (TPE) has been described in details in previous works. ${ }^{28,29,37}$ Briefly, the light source for the HRS and TPEF measurements was a modelocked femtosecond $\mathrm{Ti}$ : sapphire laser delivering at the fundamental wavelength pulses with a duration of about $140 \mathrm{fs}$ at a repetition rate of $80 \mathrm{MHz}$. After passing through a low-pass 
filter to remove any unwanted harmonic light generated prior to the cell, the fundamental beam of about $300 \mathrm{~mW}$ was focused by a low numerical aperture microscope objective into a $0.5 \mathrm{~cm}$ spectrophotometric cell containing the aqueous solutions. The HRS and two-photon emission fluorescence (TPEF) light were collected at an angle of $90^{\circ}$ from the incident direction by a $2.5 \mathrm{~cm}$ focal length lens. The HRS light was separated from the excitation light by a high-pass filter and a monochromator set at the second harmonic wavelength. The HRS light was then detected with a photomultiplier tube working in the single photon counting regime.

For the TPEF signal, the wavelength of the spectrometer (JobinYvon, iHR320 spectrometer) was scanned between $350 \mathrm{~nm}$ and $750 \mathrm{~nm}$ but the same detection unit was used. For the twophoton excited fluorescence cross section, $\sigma_{T P E F}$, the ratio of the integrated fluorescence intensities (I) for the reference and studied samples can be expressed as:

$$
\sigma_{T P E F}=\frac{\eta^{r e f} \sigma_{2}^{r e f} c^{r e f}}{c} \frac{I}{I^{r e f}}
$$

Here, the index ref denotes values related to the reference measurements. $c$ the concentration of the molecules, $\eta$ and $\sigma_{2}$ are the fluorescence efficiency and two-photon absorption cross section. For reference materials, we have chosen fluorescein dye. From the literature, we assume at the excitation wavelength of $780 \mathrm{~nm}: \sigma_{2}$ (fluorescein) $=33.3 \mathrm{GM}$ using a quantum yield of 0.9 and a two photon absorption crosssection of $37 \mathrm{GM} .^{38}$

The two-photon absorption cross section was probed using the P-scan (or Power-scan) technique during which the incident power is directly varied and the induced changes in the sample transmission are measured, as described in ref. ${ }^{28}$

Computational. The structural and one- and two-photon absorption (OPA and TPA) properties of model systems for goldcysteine and doped by silver supramolecular assemblies were determined using DFT, TDDFT 3940 and quadratic response approach ${ }^{41}, 42$ as implemented in the DALTON 43, 44 program package. For gold atoms and silver 19-e- relativistic effective core potential (19-e- RECP) taking into account scalar relativistic effects has been employed ${ }^{45}$. For all atoms split valence polarization (SVP) atomic basis set have been used ${ }^{46}$. Structural and optical properties (OPA, TPA) were determined using the hybrid B3LYP functional. ${ }^{47-49}$ In addition OPA and TPA spectra have been calculated using CAM-B3LYP functional showing the expected blue shift of the energy of excited states with respect to B3LYP functional (cf. Figure S13). Electron density difference between $\mathrm{S}_{1}$ and $\mathrm{S}_{0}$ presented in Figure $\mathrm{S} 14$ illustrates that the role of doped $\mathrm{Ag}$ atom is more visible in $\mathrm{Au}_{2}-\mathrm{Ag}-\mathrm{Cys}_{4}-\mathrm{CH}_{3}$ than in $\mathrm{Au}_{4}-\mathrm{Ag}-\mathrm{Cys}_{6}-\mathrm{CH}_{3}$ due to more compact subunit $\mathrm{Au}-\mathrm{Ag}-\mathrm{Au}$. In both cases Au-S interaction due to presence of cysteine is pronounced (cf. Figure S14). In order to illustrate ion-pairing of cysteines in both model systems doped by $\mathrm{Ag}$ atom, water solvent effect has been included using SMD approach (cf. Figure S12). ${ }^{51}$ Notice that in solvent blue shift of absorption obtained using CAM-B3LYP functional is also present. Quantitative comparison of model systems with experimental findings is difficult due to $100 \mathrm{~nm}$ scale system used in experiment but qualitatively model systems are useful for conceptual point of view.

\section{Conflicts of interest}

There are no conflicts to declare.

\section{Acknowledgements}

The authors would like to thank Céline Brunon from Science et Surface (www.science-et-surface.fr) for XPS spectra, Amina Bensallah-Ledoux (iLM) for CD spectra and Fabien Rossetti (iLM) for DLS measurements. The authors thank CNRS FrenchCroatian international lab (NCBA) for travel funding. This research was partially supported by the project STIM - REI, Contract Number: KK.01.1.1.01.0003, funded by the European Union through the European Regional Development Fund - the Operational Programme Competitiveness and Cohesion 20142020 (KK.01.1.1.01). V.B.K. and M.P acknowledge the Center for Advanced Computing and Modelling (CNRM) for providing computing resources of the supercomputer Bura at the University of Rijeka and SRCE at University of Zagreb Croatia as well as Prof. Miroslav Radman at MedILS and Split-Dalmatia County for support.

\section{References}

1. R. J. Puddephatt, Chemical Society Reviews, 2008, 37, 2012-2027.

2. H. Nie, M. Li, Y. Hao, X. Wang and S. X.-A. Zhang, Chemical Science, 2013, 4, 1852-1857.

3. H. Nie, M. Li, Y. Hao, X. Wang, S. Gao, P. Wang, B. Ju and S. X.-A. Zhang, RSC Advances, 2014, 4, 50521-50528.

4. H.-Y. Chang, Y.-T. Tseng, Z. Yuan, H.-L. Chou, C.-H. Chen, B.-J. Hwang, M.-C. Tsai, H.-T. Chang and C.-C. Huang, Physical Chemistry Chemical Physics, 2017, 19, 1208512093.

5. I. Russier-Antoine, F. Bertorelle, A. Kulesza, A. Soleilhac, A. Bensalah-Ledoux, S. Guy, P. Dugourd, P.-F. Brevet and R. Antoine, Progress in Natural Science: Materials International, 2016, 26, 455-460.

6. Y.-T. Tseng, H.-Y. Chang, S. G. Harroun, C.-W. Wu, S.-C. Wei, Z. Yuan, H.-L. Chou, C.-H. Chen, C.-C. Huang and H.-T. Chang, Analytical Chemistry, 2018, 90, 7283-7291.

7. B. Söptei, J. Mihály, I. C. Szigyártó, A. Wacha, C. Németh, I. Bertóti, Z. May, P. Baranyai, I. E. Sajó and A. Bóta, Colloids and Surfaces A: Physicochemical and Engineering Aspects, 2015, 470, 8-14.

8. S.-H. Cha, J.-U. Kim, K.-H. Kim and J.-C. Lee, Chemistry of Materials, 2007, 19, 6297-6303.

9. J. M. Forward, D. Bohmann, J. P. Fackler and R. J. Staples, Inorganic Chemistry, 1995, 34, 6330-6336.

10. H. Xu, Y. L. Song, X. R. Meng, H. W. Hou, M. S. Tang and Y. T. Fan, Chemical Physics, 2009, 359, 101-110.

11. X. Hong, S. Yinglin, M. Xiangru, H. Hongwei and F. Yaoting, Journal of Applied Polymer Science, 2012, 125, 682-689. 
12. I. Russier-Antoine, E. Benichou, G. Bachelier, C. Jonin and P. F. Brevet, The Journal of Physical Chemistry C, 2007, 111, 9044-9048.

13. C. Quintana, M. Morshedi, H. Wang, J. Du, M. P. Cifuentes and M. G. Humphrey, Nano Letters, 2019, DOI: 10.1021/acs.nanolett.8b03825.

14. J. Moreau, F. Lux, M. Four, J. Olesiak-Banska, K. Matczyszyn, P. Perriat, C. Frochot, P. Arnoux, O. Tillement, M. Samoc, G. Ponterini, S. Roux and G. Lemercier, Physical Chemistry Chemical Physics, 2014, 16, 14826-14833.

15. R. Antoine and V. Bonacic-Koutecky, Liganded silver and gold quantum clusters. Towards a new class of nonlinear optical nanomaterials, Springer, Cham, 2018.

16. R. Philip, P. Chantharasupawong, H. Qian, R. Jin and J. Thomas, Nano Letters, 2012, 12, 4661-4667.

17. N. Van Steerteghem, S. Van Cleuvenbergen, S. Deckers, C. Kumara, A. Dass, H. Häkkinen, K. Clays, T. Verbiest and S. Knoppe, Nanoscale, 2016, 8, 12123-12127.

18. S. Wang, X. Meng, A. Das, T. Li, Y. Song, T. Cao, X. Zhu, M. Zhu and R. Jin, Angewandte Chemie International Edition, 2014, 53, 2376-2380.

19. K. Brach, M. Waszkielewicz, J. Olesiak-Banska, M. Samoc and K. Matczyszyn, Langmuir, 2017, 33, 8993-8999.

20. J.-S. Shen, D.-H. Li, M.-B. Zhang, J. Zhou, H. Zhang and Y.B. Jiang, Langmuir, 2011, 27, 481-486.

21. T. Doussineau, A. Désert, O. Lambert, J.-C. Taveau, M. Lansalot, P. Dugourd, E. Bourgeat-Lami, S. Ravaine, E. Duguet and R. Antoine, The Journal of Physical Chemistry C, 2015, 119, 10844-10849

22. T. Doussineau, C. Mathevon, L. Altamura, C. Vendrely, P. Dugourd, V. Forge and R. Antoine, Angew. Chem. Int. Ed., 2016, 55, 2340-2344.

23. M. Loumaigne, C. Midelet, T. Doussineau, P. Dugourd, R. Antoine, M. Stamboul, A. Debarre and M. H. V. Werts, Nanoscale, 2016, 8, 6555-6570.

24. C. Zhou, C. Sun, M. Yu, Y. Qin, J. Wang, M. Kim and J. Zheng, The Journal of Physical Chemistry C, 2010, 114, 7727-7732.

25. F. Bertorelle, C. Moulin, A. Soleilhac, C. Comby-Zerbino, P. Dugourd, I. Russier-Antoine, P.-F. Brevet and R. Antoine, ChemPhysChem, 2018, 19, 165-168.

26. R. Randazzo, A. Di Mauro, A. D’Urso, G. C. Messina, G. Compagnini, V. Villari, N. Micali, R. Purrello and M. E. Fragalà, The Journal of Physical Chemistry B, 2015, 119, 4898-4904.

27. F. Bertorelle, I. Russier-Antoine, N. Calin, C. CombyZerbino, A. Bensalah-Ledoux, S. Guy, P. Dugourd, P.-F. Brevet, Ž. Sanader, M. Krstić, V. Bonačić-Koutecký and R. Antoine, The Journal of Physical Chemistry Letters, 2017, 8, 1979-1985.

28. I. Russier-Antoine, F. Bertorelle, N. Calin, Z. Sanader, M. Krstic, C. Comby-Zerbino, P. Dugourd, P.-F. Brevet, V. Bonacic-Koutecky and R. Antoine, Nanoscale, 2017, 9, 1221-1228.

29. I. Russier-Antoine, F. Bertorelle, M. Vojkovic, D. Rayane, E. Salmon, C. Jonin, P. Dugourd, R. Antoine and P.-F. Brevet, Nanoscale, 2014, 6, 13572-13578.

30. J. Duboisset, G. Matar, I. Russier-Antoine, E. Benichou, G. Bachelier, C. Jonin, D. Ficheux, F. Besson and P. F. Brevet, J. Phys. Chem. B, 2010, 114, 13861-13865.

31. P. N. Day, K. A. Nguyen and R. Pachter, J. Chem. Theory Comput., 2010, 6, 2809-2821.
32. C. Dai, Y. Hao, Y. Yu, M. Li and S. X.-A. Zhang, The Journal of Physical Chemistry A, 2018, 122, 5089-5097.

33. C. A. Lutomski, N. A. Lyktey, Z. Zhao, E. E. Pierson, A. Zlotnick and M. F. Jarrold, Journal of the American Chemical Society, 2017, 139, 16932-16938.

34. J. Pansieri, M. A. Halim, C. Vendrely, M. Dumoulin, F. Legrand, M. M. Sallanon, S. Chierici, S. Denti, X. Dagany, P. Dugourd, C. Marquette, R. Antoine and V. Forge, Chemical Science, 2018, 9, 2791-2796.

35. M. A. Halim, F. Bertorelle, T. Doussineau and R. Antoine, Rapid Communications in Mass Spectrometry, 2018, https://doi.org/10.1002/rcm.8204.

36. S. Guy, A. Stoita-Crisan, A. Bensalah-Ledoux, T. Vautey and L. Guy, Optical Materials, 2011, 34, 347-350.

37. I. Russier-Antoine, F. Bertorelle, R. Hamouda, D. Rayane, P. Dugourd, Z. Sanader, V. Bonacic-Koutecky, P.-F. Brevet and R. Antoine, Nanoscale, 2016, 8, 2892-2898.

38. M. A. Albota, C. Xu and W. W. Webb, Applied Optics, $1998,37,7352-7356$.

39. V. Bonačić-Koutecký, A. Kulesza, L. Gell, R. Mitrić, R. Antoine, F. Bertorelle, R. Hamouda, D. Rayane, M. Broyer, T. Tabarin and P. Dugourd, Physical Chemistry Chemical Physics, 2012, 14, 9282-9290.

40. F. Bertorelle, R. Hamouda, D. Rayane, M. Broyer, R. Antoine, P. Dugourd, L. Gell, A. Kulesza, R. Mitric and V. Bonacic-Koutecky, Nanoscale, 2013, 5, 5637-5643.

41. P. Norman, Physical Chemistry Chemical Physics, 2011, 13, 20519-20535.

42. N. H. List, R. Zaleśny, N. A. Murugan, J. Kongsted, W. Bartkowiak and H. Ågren, Journal of Chemical Theory and Computation, 2015, 11, 4182-4188.

43. Dalton, a molecular electronic structure program, Release v2016.2 (2017), see http://daltonprogram.org.

44. K. Aidas, C. Angeli, K. L. Bak, V. Bakken, R. Bast, L. Boman, O. Christiansen, R. Cimiraglia, S. Coriani, P. Dahle, E. K. Dalskov, U. Ekström, T. Enevoldsen, J. J. Eriksen, P. Ettenhuber, B. Fernández, L. Ferrighi, H. Fliegl, L. Frediani, K. Hald, A. Halkier, C. Hättig, H. Heiberg, T. Helgaker, A. C. Hennum, H. Hettema, E. Hjertenæs, S. Høst, I.-M. Høyvik, M. F. Iozzi, B. Jansik, H. J. Aa. Jensen, D. Jonsson, P. Jørgensen, J. Kauczor, S. Kirpekar, T. Kjærgaard, W. Klopper, S. Knecht, R. Kobayashi, H. Koch, J. Kongsted, A. Krapp, K. Kristensen, A. Ligabue, O. B. Lutnæs, J. I. Melo, K. V. Mikkelsen, R. H. Myhre, C. Neiss, C. B. Nielsen, P. Norman, J. Olsen, J. M. H. Olsen, A. Osted, M. J. Packer, F. Pawlowski, T. B. Pedersen, P. F. Provasi, S. Reine, Z. Rinkevicius, T. A. Ruden, K. Ruud, V. Rybkin, P. Salek, C. C. M. Samson, A. Sánchez de Merás, T. Saue, S. P. A. Sauer, B. Schimmelpfennig, K. Sneskov, A. H. Steindal, K. O. Sylvester-Hvid, P. R. Taylor, A. M. Teale, E. I. Tellgren, D. P. Tew, A. J. Thorvaldsen, L. Thøgersen, O. Vahtras, M. A. Watson, D. J. D. Wilson, M. Ziolkowski, and H. Ågren, "The Dalton quantum chemistry program system", WIREs Comput. Mol. Sci. 2014, 4:269-284 (doi: 10.1002/wcms.1172).

45. D. Andrae, U. Häußermann, M. Dolg, H. Stoll and H. Preuß, Theoretica chimica acta, 1990, 77, 123-141.

46. F. Weigend and R. Ahlrichs, Physical Chemistry Chemical Physics, 2005, 7, 3297-3305.

47. A. D. Becke, Physical Review A, 1988, 38, 3098-3100.

48. A. D. Becke, The Journal of Chemical Physics, 1993, 98, 1372-1377. 


\section{ARTICLE}

49. C. Lee, W. Yang and R. G. Parr, Physical Review B, 1988,

37, 785-789.

50. T. Yanai, D. P. Tew and N. C. Handy, Chemical Physics Letters, 2004, 393, 51-57.

51. A. V. Marenich, C. J. Cramer and D. G. Truhlar, The Journal of Physical Chemistry B, 2009, 113, 6378-6396. 